\title{
FAILURE OF THE DENJOY THEOREM FOR QUASIREGULAR MAPS IN DIMENSION $n \geq 3$
}

\author{
ILKKA HOLOPAINEN AND SEPPO RICKMAN \\ (Communicated by Albert Baernstein II)
}

\begin{abstract}
In 1929 L. V. Ahlfors proved the Denjoy conjecture which states that the order of an entire holomorphic function of the plane must be at least $k$ if the map has at least $2 k$ finite asymptotic values. In this paper, we prove that the Denjoy theorem has no counterpart in the classical form for quasiregular maps in dimensions $n \geq 3$. We construct a quasiregular map of $\mathbb{R}^{n}, n \geq 3$, with a bounded order but with infinitely many asymptotic limits. Our method also gives a new construction for a counterexample of Lindelöf's theorem for quasiregular maps of $B^{n}, n \geq 3$.
\end{abstract}

\section{INTRODUCTION}

A continuous map $f: G \rightarrow \mathbb{R}^{n}$ of a domain $G$ in $\mathbb{R}^{n}, n \geq 2$, is called quasiregular (qr) if

$$
f \in W_{n, \mathrm{loc}}^{1}(G)
$$

and there exists $K, 1 \leq K<\infty$, such that

$$
\left|f^{\prime}(x)\right|^{n} \leq K J_{f}(x) \quad \text { a.e. }
$$

The condition (1.1) means that for all $D \Subset G$ the coordinate functions of $f$ belong to the Sobolev space $W_{n}^{1}(D)$ of functions in $L^{n}(D)$ whose distributional first order partial derivatives are also $L^{n}$ integrable in $D$. In the above definition $f^{\prime}(x)$ is the formal derivative of $f$ at $x$ defined by means of the partial derivatives, $\left|f^{\prime}(x)\right|$ is the operator norm of $f^{\prime}(x)$, and $J_{f}(x)$ is the Jacobian determinant of $f$ at $x$. In this article we call a qr map $K$-quasiregular if (1.2) is satisfied. The definition extends immediately to the case $f: M \rightarrow N$ where $M$ and $N$ are connected oriented Riemannian $n$-manifolds. A quasiregular homeomorphism is by definition quasiconformal. For properties of qr maps we refer to books [4] by Yu. G. Reshetnyak, [8] by M. Vuorinen, and [6] by the second author.

Many geometric properties of analytic functions of one complex variable have their counterparts in the theory of quasiregular maps in the Euclidean space. For

Received by the editors May 2, 1994 and, in revised form, November 18, 1994.

1991 Mathematics Subject Classification. Primary 30C65.

Key words and phrases. Quasiregular maps, Denjoy theorem, Lindelöf's theorem.

Supported in part by the EU HCM contract No. CHRX-CT92-0071. 
example, Picard type theorems on omitted values are known as well as value distribution in the spirit of Ahlfors' theory of covering surfaces; see [6].

In 1907 A. Denjoy conjectured that if an entire complex analytic function has at least $2 k$ finite asymptotic values, then the order must be at least $k$. In 1921 T. Carleman proved a weaker form where $2 k$ is replaced by $5 k$. Finally, in 1929 L. V. Ahlfors [1] settled the sharp result. The sharp result for qr maps in the plane was proved by J. Jenkins [3].

It has been an open problem for some time to determine the situation of the relationship between asymptotic values and order for quasiregular maps in space. If $f: \mathbb{R}^{n} \rightarrow \mathbb{R}^{n}$ is a nonconstant quasiregular map, we define the order $\mu_{f}$ and lower order $\lambda_{f}$ of $f$ by

$$
\begin{aligned}
& \mu_{f}=\limsup _{r \rightarrow \infty}(n-1) \frac{\log \log M(r)}{\log r}, \\
& \lambda_{f}=\liminf _{r \rightarrow \infty}(n-1) \frac{\log \log M(r)}{\log r},
\end{aligned}
$$

where

$$
M(r)=\sup _{|x|=r}|f(x)| .
$$

It was proved in [7] that if $f: \mathbb{R}^{n} \rightarrow \mathbb{R}^{n}$ is a nonconstant $K$-quasiregular map with at least one asymptotic value in $\mathbb{R}^{n}$, then the lower order satisfies

$$
\lambda_{f} \geq c(n, K)>0 .
$$

This result follows also from arguments in [2].

The purpose of this paper is to show that for dimensions $n>2$ there is no lower bound for the order that tends to $\infty$ as the number of asymptotic values grows to $\infty$. More precisely, we will prove the following theorem.

1.3. Theorem. For each $n>2$ there is a quasiregular map $f: \mathbb{R}^{n} \rightarrow \mathbb{R}^{n}$ such that $\mu_{f} \leq 1$ and $f$ has infinitely many asymptotic values.

The method also gives a new proof of the counterexample to Lindelöf's theorem presented originally in [5]. This is described in Section 3.

\section{Proof of Theorem 1.3}

We shall give the proof for dimension $n=3$. The method extends to higher dimensions in a straightforward manner. The construction of the map $f$ in Theorem 1.3 is based on a modification of the Zorich map. We refer to [6, p. 15] for the usual Zorich map. To start the construction of $f$, we fix a triangulation of $\mathbb{R}^{2}=\mathbb{R}^{2} \times\{0\} \subset \mathbb{R}^{3}$ by translating the closed triangles $A_{i}, i=1, \ldots, 4$, in Figure 1 by $x \mapsto x+(2 p, 2 q), p, q \in \mathbb{Z}$. We call the set of 2 -simplexes $M^{2}$. Let $a_{i}, i=1,2, \ldots$, be distinct points of $B^{3}(1 / 2) \cap \mathbb{R}^{2}$. We choose a sequence $D_{1}, D_{2}, \ldots$ of sectors in $\mathbb{R}^{2}$ such that their mutual distances satisfy $d\left(D_{i}, D_{j}\right) \geq 8$. Each $a_{i}$ will be an asymptotic value of $f$ along the axis of $D_{i}$.

Let $z: \mathbb{R}^{3} \rightarrow \mathbb{R}^{3} \backslash\{0\}$ be a Zorich type map such that each cylinder (int $A$ ) $\times$ $\mathbb{R}^{1}, A \in M^{2}$, is mapped onto either $\mathbb{H}_{+}=\left\{x \in \mathbb{R}^{3}: x_{3}>0\right\}$ or $\mathbb{H}_{-}=\left\{x \in \mathbb{R}^{3}\right.$ : 


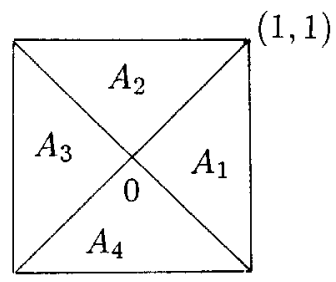

FiguRE 1

$\left.x_{3}<0\right\}$ and that $|z(x)|=e^{x_{3}}$. More precisely, each (closed) triangle $A^{c}=\{x \in$ $\left.\mathbb{R}^{3}:\left(x_{1}, x_{2}\right) \in A, x_{3}=c\right\}, A \in M^{2}$, is mapped onto either the hemisphere $S_{+}^{2}\left(e^{c}\right)$ or $S_{-}^{2}\left(e^{c}\right)$. If $z A^{c}=S_{+}^{2}\left(e^{c}\right)$, a triangle next to $A^{c}$ in the plane $x_{3}=c$ is mapped onto $S_{-}^{2}\left(e^{c}\right)$.

Next we define a function $h: \mathbb{R}^{2} \rightarrow \mathbb{R}^{1}$ by

$$
h(x)= \begin{cases}0, & \text { if } x \in \mathbb{R}^{2} \backslash \bigcup_{i} D_{i} \\ -\frac{1}{2} d\left(x, \partial D_{i}\right), & \text { if } x \in D_{i}\end{cases}
$$

where $d\left(x, \partial D_{i}\right)$ is the distance of $x$ to the boundary of $D_{i}$. Let $G \subset \mathbb{R}^{3}$ be the domain $\left\{x \in \mathbb{R}^{3}: x_{3}>h\left(x_{1}, x_{2}\right)\right\}$. We define a map $g: \bar{G} \rightarrow \mathbb{R}^{3}$ as follows. In $\overline{\mathbb{H}}_{+}$, we set $g=z$. We want that $g(x) \rightarrow a_{i}$ as $x_{3} \rightarrow-\infty$ in the component of $G \backslash \mathbb{H}_{+}$ corresponding to $D_{i}$. Call this component $G_{i}$. Let $T_{i}: \mathbb{R}^{3} \rightarrow \mathbb{R}^{3}$ be the bilipschitz map with the properties that $T_{i}=$ id in $\mathbb{R}^{3} \backslash B^{3}, T_{i}(0)=a_{i}$, and $T_{i}$ maps each line segment $[0, x]$, where $x \in S^{2}$, linearly onto the line segment $\left[a_{i}, x\right]$. We set $g=T_{i} \circ z$ in $G_{i}$. In fact, $g=T_{i} \circ z$ in $\bar{G}$ for each $i$ since $z G_{i}=B^{3} \backslash\{0\}$ and the $G_{i}$ are disjoint. Furthermore, $g$ maps the boundary $\partial A \times \mathbb{R}^{1}$ of each cylinder into the plane since $z$ does so and $T_{i} \mathbb{R}^{2}=\mathbb{R}^{2}$.

Let $F_{1}: \mathbb{R}^{3} \rightarrow \mathbb{R}^{3}$ be a map $F_{1}(x)=\left(x_{1}, x_{2}, x_{3}-h\left(x_{1}, x_{2}\right)\right)$. Clearly $F_{1}$ is bilipschitz and maps $G$ onto $\mathbb{H}_{+}$. Let $F_{2}$ be a map of the cylinder $C_{0}=\left(A_{1} \cup\right.$ $\left.\cdots \cup A_{4}\right) \times[0, \infty[$ obtained by lifting the ray $\{0\} \times[0, \infty[$ by $x \mapsto x+(0,0,1)$ and extending to each $A_{i} \times\left[0, \infty\left[\right.\right.$ linearly such that $F_{2}=$ id on $\partial\left(A_{1} \cup \cdots \cup A_{4}\right) \times \mathbb{R}^{1}$. Thus $F_{2}(x)=\left(x_{1}, x_{2}, x_{3}+1-\max \left\{\left|x_{1}\right|,\left|x_{2}\right|\right\}\right)$. We extend $F_{2}$ to $\overline{\mathbb{H}}_{+}$by conjugating with $x \mapsto x+(2 p, 2 q, 0), p, q \in \mathbb{Z}$, and call the extended map $F_{2}$, too.

We set $C_{p, q}=C_{0}+(2 p, 2 q, 0)$. Then $f_{1}=g \circ F_{1}^{-1} \circ F_{2}^{-1} \mid F_{2} \overline{\mathbb{H}}_{+}$maps each boundary part $\left(\partial F_{2} \mathbb{H}_{+}\right) \cap F_{2} C_{p, q}$ either (a) 2 to 1 onto the unit sphere $S^{2}$ if $C_{p, q} \cap$ $\left(\bigcup D_{i}\right)=\emptyset$, or (b) onto a union of 4 topological half spheres if $C_{p, q} \cap\left(\bigcup D_{i}\right) \neq \emptyset$. In the case (b), the diameter of the image is approximately $\exp \left(-d\left(C_{p, q}, \partial\left(\bigcup D_{i}\right)\right)\right)$.

Next we extend $f_{1}$ to $\overline{\mathbb{H}}_{+}$. Let $A_{i}^{p, q}=A_{i}+(2 p, 2 q)$, where $A_{i}$ is as in Figure 1 , and let $U_{i}^{p, q}$ be the part of $\mathbb{H}_{+} \backslash F_{2} \mathbb{H}_{+}$whose vertical projection on $\mathbb{R}^{2}$ is $A_{i}^{p, q}$. Thus $U_{i}^{p, q}$ is a tedrahedron whose base is $A_{i}^{p, q}$ (Figure 2).

Consider first the case (a). Then $f_{1}$ maps the 2 -simplex $a b c$ onto a half-sphere of $S^{2}$, say $S_{+}^{2}$, such that the boundary of $a b c$ is mapped onto the unit circle $S^{1} \subset \mathbb{R}^{2}$. We extend $f_{1}$ to $\bar{U}_{i}^{p, q}$ by mapping $\bar{U}_{i}^{p, q}$ onto $\bar{B}_{+}^{3}$ such that the other faces of $U_{i}^{p, q}$ are mapped into $\mathbb{R}^{2}$ and that $d \mapsto 0$. A tedrahedron next to $U_{i}^{p, q}$ is mapped onto $\bar{B}_{-}^{3}$ in a similar manner.

In the case (b) we do a natural modification of this. Now $f_{1}$ maps the 2 -simplex $a b c$ onto a topological half sphere, call this $S$, and $f_{1}$ is extended to $\bar{U}_{i}^{p, q}$ as above 


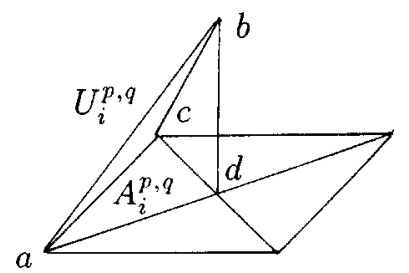

FIGURE 2

by mapping $\bar{U}_{i}^{p, q}$ onto the closed topological ball bounded by $S$ and a part of $\mathbb{R}^{2}$ such that $d \mapsto a_{i}$.

We obtain an extended map $f_{1}: \overline{\mathbb{H}}_{+} \rightarrow \mathbb{R}^{3}$ with the properties that $f_{1} \mathbb{R}^{2}=\mathbb{R}^{2}$ and that $f_{1}(x) \rightarrow a_{i}$ as $x \rightarrow \infty$ along the axis of the sector $D_{i}$.

Finally, we define $f: \mathbb{R}^{3} \rightarrow \mathbb{R}^{3}$ by

$$
f(x)= \begin{cases}f_{1}(x), & \text { if } x \in \overline{\mathbb{H}}_{+} \\ \bar{f}_{1}(\bar{x}), & \text { if } x \in \mathbb{H}_{-},\end{cases}
$$

where $\bar{x}=\left(x_{1}, x_{2},-x_{3}\right)$ is the reflection of $x=\left(x_{1}, x_{2}, x_{3}\right)$ in $\mathbb{R}^{2}$. Then $f$ has the desired properties of Theorem 1.3.

\section{A COUNTEREXAMPle to LindelÖF'S THEOREM}

In this section we shall show that the method from Section 2 also gives a new counterexample to Lindelöf's theorem for quasiregular maps of $B^{n}$ for $n \geq 3$. Such an example was originally given in [5] (see also [6, p. 193]) and the statement is formulated as follows.

3.1. Theorem. For each $n \geq 3$ there exist a bounded qr mapping $f$ of $B^{n}$ and $a$ point $b \in \partial B^{n}$ such that $f$ has infinitely many asymptotic values at $b$ and no angular limit at $b$.

Proof. Also now the proof is given for $n=3$ and it extends to other dimensions easily. We again identify $\mathbb{R}^{2}=\mathbb{R}^{2} \times\{0\} \subset \mathbb{R}^{3}$ and set

$$
\begin{aligned}
X & =[0,1] \times] 0,1] \subset \mathbb{R}^{2}, \\
A^{*} & =\left\{x \in \mathbb{R}^{3}:\left(x_{1}, x_{2}\right) \in A,-x_{2} \leq x_{3} \leq x_{2}\right\} \text { if } A \subset X .
\end{aligned}
$$

We shall construct a quasiregular map of $V=\operatorname{int} X^{*}$ with infinitely many asymptotic values at $0 \in \partial X^{*}$. Since $V$ is bilipschitz equivalent to $B^{n}$, the first statement of Theorem 3.1 follows. We start out with a dyadic Whitney type subdivision of $X$ into squares shown in Figure 3. For $k=1,2, \ldots$ set

$$
\begin{aligned}
& Z_{0}=\left\{x \in X:\left(\frac{x_{1}}{4}\right)^{2} \leq x_{2}\right\} \\
& X_{k}=\left\{x \in X: \frac{1}{2}\left(\frac{x_{1}}{4}\right)^{4 k-2} \leq x_{2} \leq\left(\frac{x_{1}}{4}\right)^{4 k-2}\right\}, \\
& Y_{k}=\left\{x \in X:\left(\frac{x_{1}}{4}\right)^{4 k} \leq x_{2} \leq \frac{1}{2}\left(\frac{x_{1}}{4}\right)^{4 k-2}\right\}, \\
& Z_{k}=\left\{x \in X:\left(\frac{x_{1}}{4}\right)^{4 k+2} \leq x_{2} \leq\left(\frac{x_{1}}{4}\right)^{4 k}\right\} .
\end{aligned}
$$




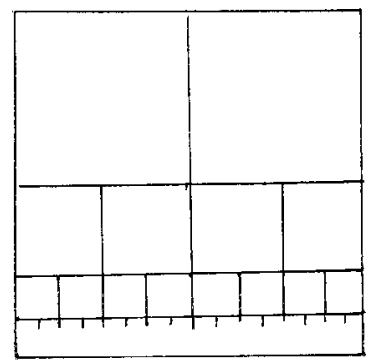

FiguRE 3

We modify the subdivision of Figure 3 as follows. For $k \geq 1$ let $\psi_{k}: X \rightarrow X$ be the map of the form $\psi_{k}\left(x_{1}, x_{2}\right)=\left(x_{1}, a_{k}\left(x_{1}\right) x_{2}+b_{k}\left(x_{1}\right)\right)$, where $a_{k}$ and $b_{k}$ are functions such that $\psi_{k}$ keeps $\partial X_{k} \cap \partial Z_{k-1}$ fixed and moves $\partial Y_{k} \cap \partial Z_{k}$ onto $\partial X_{k} \cap \partial Y_{k}$. For $k \geq 0$ let $Z_{k}^{\prime}$ be the union of the (closed) squares of Figure 3 that are contained in $Z_{k}$. In our modification the sets $Z_{k}^{\prime}$ are kept unchanged. Let $W_{k} \subset X$ be the closed part between $Z_{k-1}$ and $Z_{k}^{\prime}$. Write $\psi_{k}\left(W_{k}\right)=\tilde{W}_{k}$. This means that the squares touching $X_{k} \cup Y_{k}$ are squeezed upwards. Then we fill the part $W_{k} \backslash \tilde{W}_{k}$ as follows. Each square $Q$ in $Z_{k}^{\prime}$ touching $W_{k}$ defines a column $\hat{Q}=\left\{x \in W_{k} \backslash \tilde{W}_{k}: x_{1} \in \pi_{1} Q\right\}$, where $\pi_{1}\left(x_{1}, x_{2}\right)=x_{1}$. Let the side length of $Q$ be $a$. We fill the column $\hat{Q}$ by squares congruent to $Q$ plus a leftover part nearest to $\tilde{W}_{k}$ whose smaller vertical side length $s$ is required to satisfy $s \in[a, 2 a[$.

Next we triangulate this new subdivision of $X$ by joining vertices by line segments and without adding any new vertices. Call the simplicial $2-$ complex $P^{\prime}$ with space $X$. Finally we perform the geometric barycentric subdivision of $P^{\prime}$ and obtain a 2 -complex $P$. The complex $P$ has the property that each vertex in int $X$ belongs to an even number of 2 -simplices; we let $P^{2}$ be the 2 -simplices of $P$.

To construct the required map $f$ of $V$ we apply the method from Section 2 as follows. Each group of four triangles $A_{i}, i=1, \ldots, 4$ (Figure 1), with their translations by $x \mapsto x+(2 p, 2 q), p, q \in \mathbb{Z}$, corresponds now to one 2-simplex in $P^{\prime}$. The center in Figure 1 and its translations correspond to the barycenters of the triangulation $P^{\prime}$. We start with a Zorich-type map $\tilde{z}$ restricted to $X^{*} \cap \overline{\mathbb{H}}_{+}$. The cylinders are $A^{*} \cap \overline{\mathbb{H}}_{+}, A \in P^{2}$, and we fix $\tilde{z}$ so that $\tilde{z}$ maps the boundary part $\left\{x \in X^{*}: x_{3}=x_{2}\right\}$ onto $S^{2}(2)$ and each $A$ onto a topological half sphere whose diameter is approximately $\exp \left(-d\left(A^{*} \cap \mathbb{H}_{+}\right) / d(A)\right)$, where $d(E)$ is the diameter of a set $E$. In addition we require that $|\tilde{z}| \leq 1$ in $X$ and $|\tilde{z}|=1$ in $Z_{k}, k \geq 0$. Our map $\tilde{z}$ corresponds to $z \circ F_{1}^{-1}$ in Section 2. Then we perform shifting $\tilde{F}_{2}$ in the $x_{3}$ direction similar to $F_{2}$ by using the barycenters for points of maximal shifts. Define $\tilde{g}$ by $\tilde{g}=T_{i} \circ \tilde{z}$ in $\left(X_{i}^{*} \cup Y_{i}^{*}\right) \cap \overline{\mathbb{H}}_{+}$, where $T_{i}$ is as in Section 2 . We extend $\tilde{f}_{1}=\tilde{g} \circ \tilde{F}_{2}^{-1} \mid \tilde{F}_{2}\left(X^{*} \cap \mathbb{H}_{+}\right)$similarly as before. Let $A^{\prime}$ be a 2-simplex in $P^{\prime}$. We divide the treatment again into two cases: (a) if $A^{\prime}$ does not meet any $X_{i} \cup Y_{i}, i \geq 1$, we map the barycenter of $A^{\prime}$ to 0 ; (b) if $A^{\prime} \cap\left(X_{i} \cup Y_{i}\right) \neq \emptyset$, the barycenter is mapped to $a_{i}$. We get a map $\tilde{f}_{1}$ of $X^{*} \cap \mathbb{H}_{+}$, which we extend by reflection to $\tilde{f}: X^{*} \rightarrow \bar{B}^{3}(2)$. The restriction $\tilde{f} \mid V$ is quasiregular and has the asymptotic limit $a_{i}$ along the path $t \mapsto\left(t, \frac{1}{2}\left(\frac{t}{4}\right)^{4 i-2}, 0\right) \in V$ as $t \rightarrow 0$. With a bilipschitz map $F: \bar{V} \rightarrow \bar{B}^{n}$ we also see that $f=\tilde{f} \circ F^{-1} \mid B^{n}$ has no angular limit at $F(0)$. Theorem 3.1 is proved. 


\section{ADDENDUM}

After this article was completed we received a manuscript "On a method of Holopainen and Rickman" by D. Drasin. He constructs an entire quasiregular map on $\mathbb{R}^{n}, n \geq 3$, of order $n-1$ with every $a \in \mathbb{R}^{n}$ asymptotic.

\section{REFERENCES}

[1] Ahlfors, L.V., Über die asymptotischen Werte der ganzen Funktionen endlichen Ordnung, Ann. Acad. Sci. Fenn. Ser. A 32;6 (1929), 1-15.

[2] Granlund, S., Lindqvist, P., Martio, O., F-harmonic measure in space, Ann. Acad. Sci. Fenn. Ser. A I Math. 7 (1982), 233-247. MR 84f:30031

[3] Jenkins, J., On the Denjoy conjecture, Canad. J. Math. 10 (1958), 627-631. MR 20:5849

[4] Reshetnyak, Yu. G., Space mappings with bounded distortion, Translations of Mathematical Monographs 73, American Mathematical Society, Providence, RI, 1989. MR 90d:30067

[5] Rickman, S., Asymptotic values and angular limits of quasiregular mappings of a ball, Ann. Acad. Sci. Fenn. Ser. A I Math. 5 (1980), 185-196. MR 82b:30019

[6] Rickman, S., Quasiregular mappings, Ergebnisse der Mathematik und ihrer Grenzgebiete, Band 26, Springer-Verlag, Berlin Heidelberg New York, 1993. CMP 94:01

[7] Rickman, S., Vuorinen, M., On the order of quasiregular mappings, Ann. Acad. Sci. Fenn. Ser. A I Math. 7 (1982), 221-231. MR 85g:30029

[8] Vuorinen, M., Conformal geometry and quasiregular mappings, Lecture Notes in Math. 1319, Springer - Verlag, Berlin Heidelberg New York, 1988. MR 89k:30021

Department of Mathematics, P.O. Box 4 (Hallituskatu 15), Fin-00014 University of Helsinki, Finland

E-mail address: ih@geom.helsinki.fi 